\title{
Medical Image of the Week: Levamisole-Induced Vasculitis
}

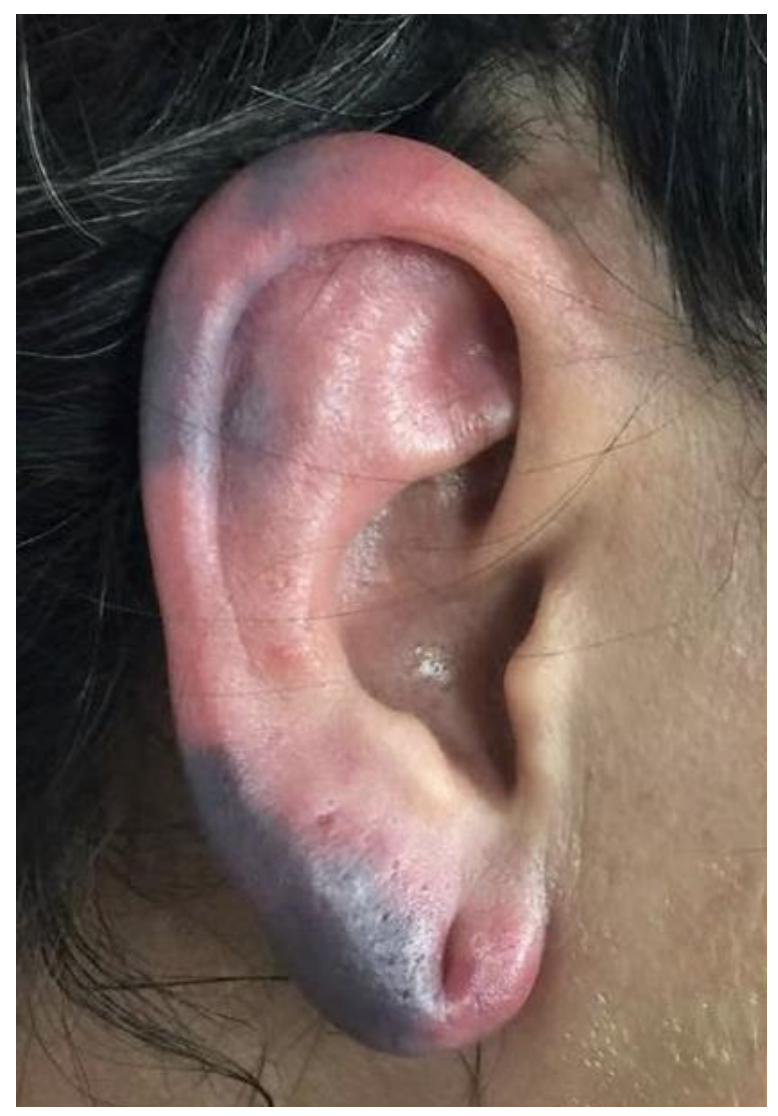

Figure 1. Purpuric lesion on the auricle of the ear.

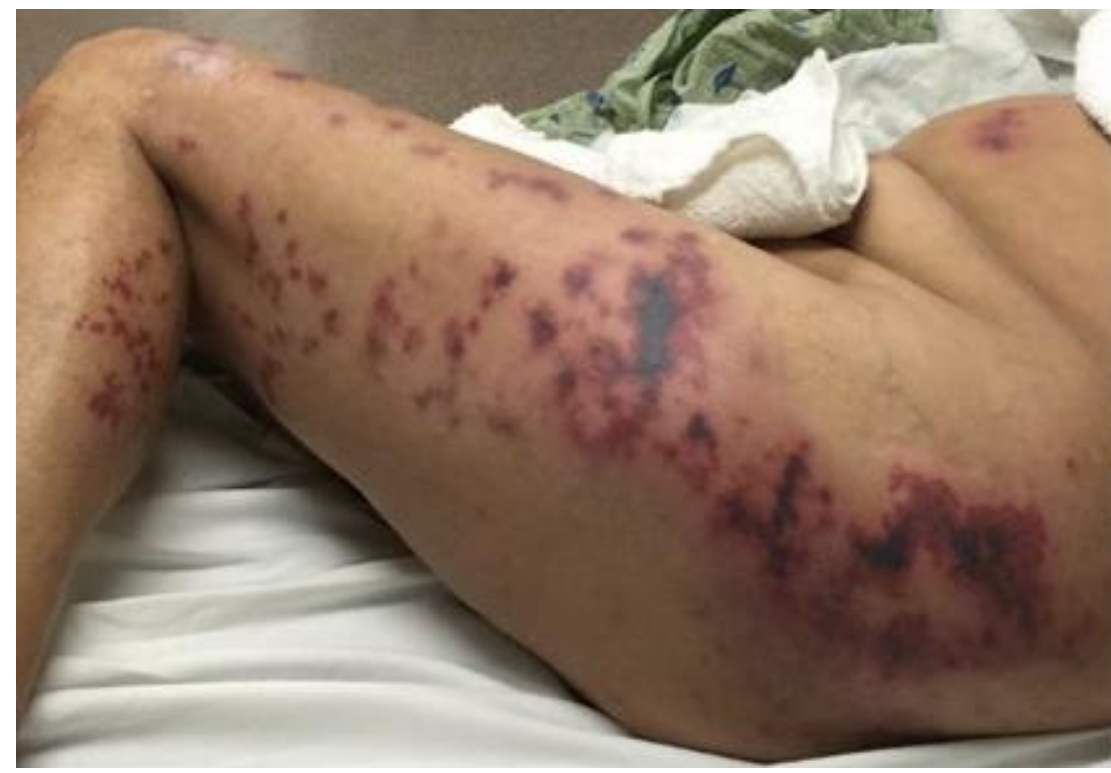

Figure 2. Diffuse purpuric lesions in retiform patterns of the trunk and extremities. 
A 51-year-old Hispanic woman presented with a worsening, diffuse, painful purpuric rash over the last 3 months, involving both auricles of the ears, both lower extremities, and her trunk. She also reported purulent discharge drainage from one of the lesions on her right posterior thigh, associated with fever and chills for one day. She was a daily cocaine user for the last 30 years.

On examination, she was febrile and tachycardic. There were diffuse retiform like non-blanching purpuric lesions without necrosis on ears, her lower extremities, and trunk (Figure 1 and 2). There was an open wound on the posterior aspect of her right thigh that had purulent drainage.

Laboratory investigations revealed neutropenia, normal complete metabolic panel, high erythrocyte sedimentation rate (ESR) and high C-reactive protein. Further autoimmune work-up revealed positive perinuclear antineutrophil cytoplasmic antibodies (p-ANCA), high anti-myeloperoxidase antibodies (MPO) titer, elevated IgM anticardiolipin antibodies, negative antinuclear antibodies (ANA), and low complement levels. HIV, hepatitis viral panel and cryoglobulin were negative. Urine toxicology was positive for cocaine. Benzoylecgonine, $\mathrm{m}$ $\mathrm{OH}$-benzoylecgonine, and cocaethylene, which are cocaine metabolites were detected using qualitative liquid chromtaography-mass spectrography.

Her presentation is suggestive of drug-induced vasculitis, likely secondary to levamisole-adulterated cocaine, complicated by an abscess. We started intravenous (IV) vancomycin and performed a bedside incision and drainage for the abscess on her posterior thigh. On the third day of hospital admission, all her lesions improved remarkably with abstinence from cocaine.

Levamisole, an atihelminthic agent, is used as treatment for autoimmune disorders and cancer due to its immunomodulating effects. Association between levamisole and cutaneous vasculitis was first described in 1978 in a patient who has rheumatoid arthritis treated with levamisole (1). In 2000, the Food and Drug Administration (FDA) banned the use of levamisole due to its side effects profile. However, since 2009, reports of agranulocytosis and vasculitis associated with levamisole have been increasing. The emergence of these cases is attributed by the increased contamination of cocaine with levamisole. More than $70 \%$ of cocaine found in North America contains levamisole $(2,3)$.

Clinical features of levamisole-induced vasculitis include retiform purpura or purpura that has an angulated or branched configuration. Some patients may develop bacterial superinfection of the purpuric lesions, which was seen in our patient. This requires more attention to prevent further complications as these patients are immune suppressed. Two classic pathologic findings of these rashes are leukocytoclastic vasculitis of small vessels with fibrinoid necrosis of the wall of the vessels and formation of fibrin thrombi in the small vessels of superficial and deep dermis (4). These individuals commonly have neutropenia, positive ANCA serology, and elevated anti-MPO antibody titers (5). 
Treatment includes supportive measures and abstinence from cocaine. Due to its increasing incidence, physician should be made aware of this disease entity due to its life threatening complications - neutropenia.

Kai Rou Tey, MD ; Enrique Villavicencio, $\mathrm{MD}^{2}$; and Don Leo Pepito, $\mathrm{MD}^{1}$

${ }^{1}$ Department of Internal Medicine,

${ }^{2}$ Department of Neurology

University of Arizona College of Medicine-South Campus

Tucson, AZ

\section{References}

1. Macfarlane DG, Bacon PA. Levamisole-induced vasculitis due to circulating immune complexes. Br Med J. 1978 Feb 18;1(6110):407-8. [CrossRef] [PubMed]

2. Centers for Disease Control and Prevention (CDC). Agranulocytosis associated with cocaine use - four States, March 2008-November 2009. MMWR Morb Mortal Wkly Rep. 2009 Dec 18;58(49):1381-5. [CrossRef] [PubMed]

3. Buchanan JA, Heard K, Burbach C, Wilson ML, Dart R. Prevalence of levamisole in urine toxicology screens positive for cocaine in an inner-city hospital. JAMA. 2011 Apr 27;305(16):1657-8. [CrossRef] [PubMed]

4. Roberts JA, Chévez-Barrios P. Levamisole-Induced Vasculitis: A characteristic cutaneous vasculitis associated with levamisole-adulterated cocaine. Arch Pathol Lab Med. 2015 Aug;139(8):1058-61. [CrossRef] [PubMed]

5. McGrath MM, Isakova T, Rennke HG, Mottola AM, Laliberte KA, Niles JL. Contaminated cocaine and antineutrophil cytoplasmic antibody-associated disease. Clin J Am Soc Nephrol. 2011 Dec;6(12):2799-805. [CrossRef] [PubMed] 